\section{THERMODYNAMICS OF ADSORPTION}

N informal discussion on "The Application of
Thermodynamics and Statistical Mechanics to
Adsorption Problems" was held in the Chemistry
Department, University College, Dundee (University
of St. Andrews), during July $20-21$. Twenty-five
people were present. With this small group a very
close exchange of views was possible, and the dis-
cussion throughout was brisk and lively. The
discussion was divided into two parts : (1) monolayer
adsorption, (2) multilayer adsorption and hysteresis.
The first section was opened by Dr. C. Kemball
(Cambridge), who presented a paper on the entropy
of adsorption. He showed how a knowledge of
entropy changes can throw light on the mobility and
lateral interaction of the adsorbed molecules, and
how the entropy can be obtained both from experi-
mental data and also theoretically, and stressed the
importance of resolving free energies into their
respective heat and entropy components. Two cases
were dealt with, the mobile and immobile film.
Comparing the experimental results with the theor-
eticel, it is found that the results can be divided
into four classes : 'supermobile' (xenon on mercury
at $260^{\circ} \mathrm{K}$.), mobile (ethane on chabazite at $430^{\circ} \mathrm{K}$.),
intermediate (benzene on mercury at $323^{\circ} \mathrm{K}$.) and
immobile (hydrogen on glass at $20^{\circ} \mathrm{K}$.). Dr. Kemball
stressed the need for accurate surface-area determ-
inations.

Prof. D. H. Everett (Dundee) prefaced his paper on the relation between heats and entropies of adsorption with a discussion of the various thermodynamic quantities which have been used to describe adsorption data. The conditions under which these sets of free energies, heats and entropies can be derived from experimental results were precisely specified and the relationships between them indicated. As in the study of solutions, a reference system is desirable. In monolayer adsorption this can be either an ideal localized monolayer or an ideal two-dimensional gas. Using the former model as a reference state, it has been found that the 'excess' partial molar entropy of adsorption, obtained by deducting the estimated configurational entropy from the experimental entropy, is a linear function of the differential heat of adsorption. This line, for twenty adsorbates on charcoal at $0^{\circ} \mathrm{C}$., was superposable on the Barclay-Butler heat-entropy plot for the evaporation of liquids. An extension of this method of analysis to low-temperature adsorption results (with Dr. D. M. Young) shows that the characteristics of the heat-entropy plot are markedly influenced by temperature. Prof. Everett concluded that the localized monolayer seems to be a satisfactory reference system, and pointed out the possibilities of a more extensive examination of the heat-entropy relation.

Prof. R. M. Barrer (Aberdeen) and Dr. Kemball both stressed the difficulties encountered in reaching any fundamental conclusions about heat-entropy relations, as the experimentally accessible range of $\Delta G$ values is often rather small compared with the corresponding changes in $\Delta H$ and $\Delta S$.

Dr. H. C. Longuet-Higgins (Manchester) outlined the method by which he is bringing statistical mechanics to bear on the general relation between heats and entropies. The Barclay-Butler rule for the heats and entropies of solution of a gas in a liquid can be simply derived, using Henry's law, if certain additional assumptions equivalent to the Law of Corresponding States are made regarding the nature of the solute-solvent interactions. This treatment has been extended to adsorption assuming a mobile film and Henry's law, and it was shown that here also a linear heat-entropy relation is to be expected.

Dr. G. Szasz (Office of Naval Research, American Embassy, London), speaking on calorimetric measurements of specific heats and entropies of adsorption, presented some recent experimental work from the laboratories of Prof. J. G. Aston (Pennsylvania State College) and Dr. J. A. Morrison (National Research Council, Ottawa). From the results of Aston, Szasz and Kingdon, a calculation has been made of the entropy difference at $0^{\circ} \mathrm{K}$. between a film of nitrogen on titanium dioxide corresponding to a completed monolayer, and a film containing 0.6 this amount of nitrogen. They found a positive value $(+1 \cdot 6 \pm$ 1.6 cal. deg. ${ }^{1}$ mole $^{-1}$ ) instead of the negative value expected for an ideal localized monolayer. An explanation for this result was suggested by Prof. Barrer, who pointed out that on a heterogeneous surface the entropy calculated by the usual formulæ of statistical mechanics would be in error. Later in the discussion an alternative explanation was suggested by Prof. Everett, who showed that it would be accounted for if, in fact, a second layer had started to form before the completion of the monolayer. Measurements have also been made by Dr. Morrison and Dr. Los of the specific heat at four-tenths coverage, as a function of temperature, for argon on rutile of a high specific surface down to $14^{\circ} \mathrm{K}$. Dr. Szasz presented some of their preliminary results, which exhibited a number of interesting features. Detailed discussion of these points seemed unwise until confirmed by later work.

Dr. A. R. Miller (Cambridge) discussed the theorotical calculation of heats of adsorption and showed that the variation of heat with coverage may be caused either by interactions between the adsorbed particles or by surface heterogeneity. He pointed out that no experiments of sufficient accuracy have been published which make it possible at present to distinguish between these two effects. Dr. Miller also mentioned that measured heats of adsorption of polar molecules indicate that existing methods of dealing with long-range interactions between adsorbed molecules are inadequate. He suggested that some recent work on the order-disorder problem could be extended to take account of long-range forces. The ensuing discussion dealt with uniformity and nonuniformity of surfaces. Dr. J. H. Singleton (Aberdeen) directed attention to the striking change in the surface properties of a metal when only a few molecules are chemisorbed on it. Dr. Szasz referred to recent Russian work which indicates that the change of heats with coverage can be accounted for by 'induced heterogeneity' on an initially uniform surface. Mr. A. S. Porter (Imperial College, London) mentioned the experiments of Emmett and Kummer with carbon-14 monoxide and carbon-12 monoxide, which these authors claim demonstrate the effect of heterogeneity.

Dr. D. M. Young (Dundee) showed that recent work with Dr. F. C. Tompkins (Imperial College, London) has confirmed Orr's experimental heats of adsorption on cæsium iodide crystals, but also that Orr's calculations do not refer to the same crystallographic plane as his experiments. Recent experimental work at Dundee shows that the heats of adsorption of 
argon on potassium chloride are some $500 \mathrm{cal} . / \mathrm{mole}$ higher on the (111) than on the (100) plane. A possible link between these studies and the theory of crystal growth and habit modification was indicated. Some unpublished work of S. Ross and G. E. Boyd (Oak Ridge) on the low-temperature adsorption of ethane on a number of sodium chloride preparations was mentioned. These results suggest that each step in a step-wise isotherm can be attributed to a different crystallographic plane present on the surface. Dr. Kemball directed attention to some interesting stepwise isotherms for acetone on liquid mercury, where each step appears to denote the filling of a fresh layer. The question of step-wise isotherms was discussed and it was felt that the available experimental results were insufficient to warrant a general interpretation of this phenomenon.

The section on multimolecular adsorption was introduced by Prof. Barrer (Aberdeen). Brief mention was made of the recent statistical derivation (with Dr. R. R. Fergusson (Aberdeen)) of the Hüttig equation; it was pointed out that this equation can also be derived using Hill's method of 'vertical counting'. He considered that the main weakness of the Hüttig and Brunauer-Emmett-Teller (B.E.T.) models is their neglect of interaction forces. A new statistical treatment of adsorption was outlined in which it is assumed that sorption centres initiate the growth of compact clusters of limited size (as distinct from the columnar clusters assumed in B.E.T. or Hüttig treatments). Neglecting lateral interactions between molecules in the cluster, jsotherms obtained are type I with some variations. Prof. Barrer and Mr. Robins (Aberdeen) have made considerable progress with another new model which, like Polanyi's treatment, takes into account interactions between sorbed molecules, as. well as the comparatively longrange nature of dispersion forces acting between a massive substrate and a gaseous molecule. The adsorbed phase is regarded as a compressed threedimensional gas. It is assumed that van der Waals' equation of state is valid over the entire range of the adsorption isotherm, and that the interaction energy between a molecule and the surface varies as the inverse cube of the distance. Although no simple relationships emerge, it has been possible, using selected values of the heat of adsorption, to obtain for adsorption on a plane surface isotherms corresponding to the first three types in the B.E.T. classification, all the isotherms reducing to type $I$ at temperatures above the critical temperature of the adsorbate. The theory is also being extended to include adsorption between parallel planes (cracks) and in capillaries, and on this basis it appears possible to obtain the other two general types of isotherm.

Prof. Everett indicated a possible approach to the thermodynamic treatment of multilayer adsorption. If the Barclay-Butler rule be assumed valid over the entire adsorption process, a knowledge of the differential heat of adsorption will permit the interpolation of the excess entropy term from the Barclay-Butler line. A comparison of this term with the experimental entropy would give a measure of the configurational entropy as a function of the amount adsorbed. This treatment has been applied to some results of Crawford and Tompkins; the configurational entropy corresponds closely to that calculated for an ideal localized monolayer up to about half-coverage, after which a sudden inflexion marks the beginning of $a_{0}$ steep rise in partial molar configurational entropy. The general shape of the curves obtained by this method correspond to that expected for a localized model, where adsorption in the second layer takes place on sites provided by groups of first-layer molecules. The increase in entropy arises from the increasing number of arrangements possible when adsorbed molecules can also occupy sites in the second layer.

Isotherms for a number of organic vapours on nickel oxide were presented by $\mathrm{Mr}$. G. Schreiner (Cambridge). These have been analysed from a thermodynamic point of view using Dr. Kemball's method, in which values of the entropy are calculated at a standard state corresponding to a definite fraction of the surface covered. The assignment of a definite value of the fractional coverage is only possible if reliable values of the monolayer capacity are available. To this end, a detailed examination of the values obtained from the B.E.T. and the Volmer isotherms has been made. The B.E.T. values are about seven-tenths of the Volmer values, but it was concluded that the latter lead to a more consistent definition of the standard state.

Mr. W. I. Whitton (Dundee) described some isotherms which he has measured for benzene on charcoal over a range of temperatures. At high coverages the adsorption and desorption isotherms are separated by a hysteresis loop, but the marked capillary condensation just as the saturation vapour pressure is reached was found to be completely reversible. The general shape of these isotherms suggests that capillary condensation does not become appreciable until the pressure approaches the saturation vapour pressure. The vigorous discussion of adsorption hysteresis which ensued demonstrated the need for more detailed theoretical and experimental work on this topic.

Prof. W. F. K. Wynne-Jones (Newcastle), Drs. S. J. Gregg (Exeter), P. Meares (Aberdeen), R. C. Pink (Belfast), R. R. Smith (Dundee), and Mr. F. W. Smith (Dundee) slso contributed to the discussion.

W. I. WHITTON

D. M. YOUNG

\section{INDIAN ASSOCIATION FOR THE CULTIVATION OF SCIENCE \\ ANNUAL REPORT FOR 1949-50}

$T$

HE annual general meeting of the Indian Association for the Cultivation of Science was held in Calcutta on July 25. The Joy Kissen Mookerjee Gold Medals for 1947 and 1948 were awarded to Mme. Irene Joliot-Curie, director of the Radium Institute, Paris, and to Dr. Arthur E. Morgan, president of the Tennessee Valley Authority, respectively. Sir Robert Robinson, president of the Royal Society, received the Association's Bimala Churn Law Gold Medal for 1945, and the same Medal for 1949 was awarded to Sir Alexander Fleming. Prof. Hermann E. Mark, director of the Polymer Research Institute, United States, delivered a course of lectures as Coochbehar professor of the Association.

The members of the Council of the Association for 1950-51 are as follows : President, Prof. M. N. Saha; Vice-Presidents, Dr. J. C. Ghosh and Dr. D. M. Bose; Honorary Director, Prof. P. Ray ; Ordinary Members, Dr. S. R. Bose, Sri Dwijesh Chandra Chakravarti, Dr. B. C. Guha, Dr. Satish Chandra Ganguly, Prof. S. K. Mitra, Prof. P. C. Mahanti, Prof. S. K. Ray, Hon. Sri C. C. Biswas, Dr. S. K. Banerji and Dr. 